\title{
Correlación histopatológica y ecografía de la madurez placentaria en el embarazo de alto riesgo
}

\author{
Dres.: Jaime Ferro Camargo*; Hugo Nossa Moreno*; \\ German Montoya Sánchez**; Juan G. Uribe Angel***; \\ Pilar Duque de Didomenico**** y Martha Cruz de la Torre*****
}

\section{MATERIAL Y METODOS}

Se escogieron cuarenta pacientes que concurrieron al servicio de admisión de Obstetricia en la Sala de Partos del Hospital de San José, Facultad de Medicina de la Universidad del Rosario, en los meses de octubre, noviembre y diciembre de 1982 y enero de 1983, con embarazo de 36 semanas en adelante $y$ de alto riesgo dado por enfermedad Hipertensiva del embarazo e Hipertensión arterial asociada o agregada, inclu-

* Residente Depto. de Gineco-Obstetricia. Hospital de San José. Facultad de Medicina del Rosario.

* Jefe del Depto. de Gineco-Obstetricia Hospital de San José. Facultad de Medicina.

*** Profesor Depto. de Patología. Hospital de San José, Facultad de Medicina.

**** Profesor Depto. de Radiología. Hospital de San José. Facultad de Medicina del Rosario.

**** Residente Depto. de Radiología. Hospital de San José. Facultad de Medicina del Rosario. yendo dentro del estudio dos pacientes diabéticas no hipertensas.

Las pacientes habían sido diagnosticadas unas en la consulta prenatal y otras en el momento de su ingreso al servicio de admisión de urgencias de Obstetricia, con los parámetros clínicos y paraclínicos de Clasificación dados por Chesley en 1971, donde casi todos los padecimientos hipertensivos que ocurren durante el embarazo quedan incluidos y los pequeños cambios que se les han hecho se refieren a terminología de poca variación (1).

Unas pacientes venían siendo tratadas en la consulta prenatal, otras a su ingreso en admisión de urgencias y cada una de acuerdo a su estado con: reposo, sedantes generalmente de tipo fenobarbital $y$ antihipertensivos tipo hidralazina y/o alfa metil dopa según el caso.

A todas las parturientas se le practicó a su ingreso Ecografía Obstétrica y Amniocentesis previas al parto, el cual en ningún caso fue posterior a las siguientes cuarenta y ocho horas de los dos anteriores exámenes; las placentas obtenidas fueron enviadas para revisión $y$ posterior procesamiento por anatomía patológica. 
Cuadro No. 1

CLASIFICACION DE GRANNUM YCOLS. 1979

\section{CAMBIOS}

ECOGRAFICOS

EN LA MADUREZ

PLACENTARIA

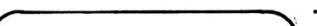

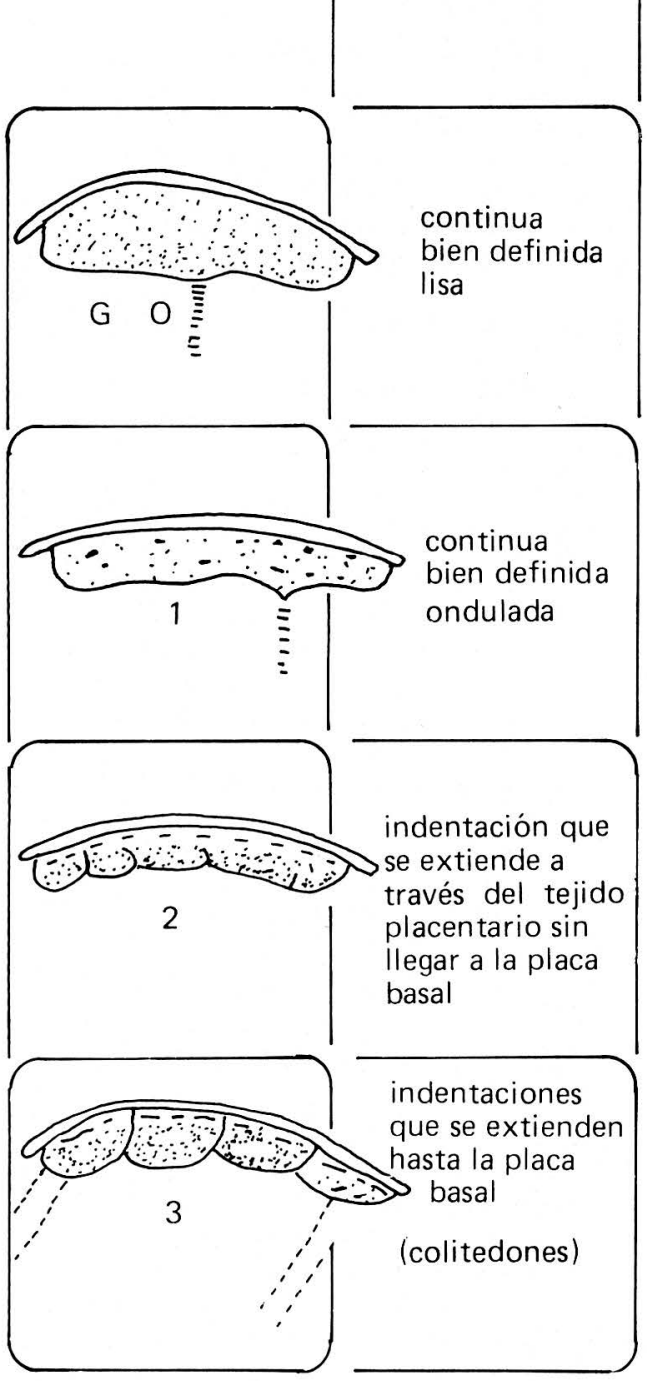

VELLOSIDADES

TEXTURA

PLACENTARIA

Grosor

promedio: $\mathrm{cms}$

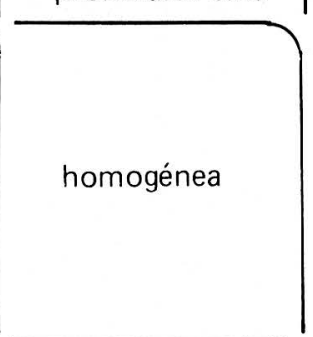

no

homogénea

áreas ecogénicas

lineales

$1.4 \mathrm{~mm}$

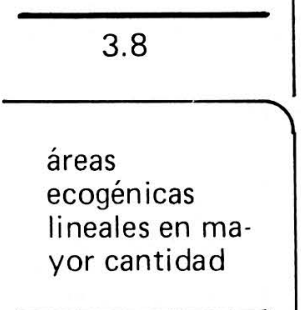

\begin{tabular}{l}
\hline \multicolumn{1}{c}{3.66} \\
\hline áreas redondeadas \\
centrales y \\
anecogénicas \\
áreas irregulares \\
periféricas y eco \\
génicas que pro- \\
ducen sombra \\
$\frac{\text { acústica (calcio) }}{3.48}$ \\
\hline
\end{tabular}

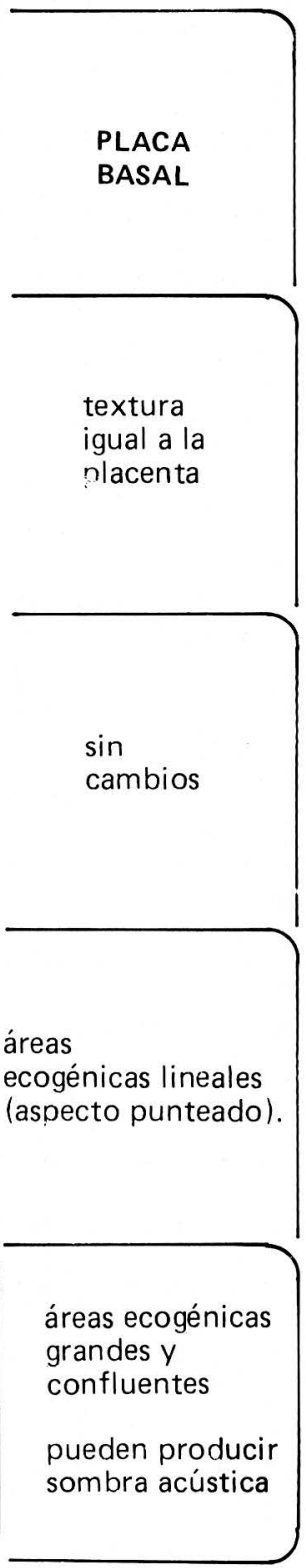


La amniocentesis fue practicada en cada paciente mediante punción suprapubica o paraumbilical inferior derecha o izquierda, de acuerdo a la ecografía, guía y control de procedimiento. Fueron obtenidos entre diez y veinte centímetros cúbicos de líquido amniótico de cada paciente, los cuales fueron estudiados físicamente en primera instancia, posteriormente se pasaron a refrigeración para ulterior análisis bioquímico de madurez, el cual resultó innecesario practicar, ya que ninguno de los recién nacidos presentó síndrome de dificultad respiratoria.

Los exámenes ecográficos se practicaron con equipo de tiempo real y estático, obteniéndose registros en placas para posteriores valoraciones. Se estudió el feto y la placenta. El feto se examinó morfológicamente al cual se le determinó el diámetro biparietal (DBP) por los parámetros ya establecidos (2) (3). En el estudio de la placenta se determinó los grados de madurez por los parámetros de clasificación de Grannumy Cols. (4), en la cual se dan cuatro grados $(0$ a III) calificados por los cambios morfológicos que se observan durante el embarazo en la placa corionica, la placa basal y la textura o substancia placentaria. (Cuadro No. 1).

Para el estudio morfológico de la placenta, se pesó el órgano y se tomaron secciones cada centímetro, con el fin de valorar lesiones macroscópicas. De cada placenta se tomaron cortes histológicos de la sección representativa del centro de la placenta. La valoración histológica se llevó a cabo, escogiendo vellosidades promedio de áreas escogidas y midiendo en las mismas el área promedio de las vellosidades, el área promedio de los capilares, el número de capilares y la relación entre el área promedio de las vellosidades $y$ de los capilares conocida con la letra $Z(5)$.

\section{RESULTADOS}

Se practicó una correlación entre el grado de maduración placentaria por Ecografía, denominada con la sigla G, y la maduración morfológica o histológica de las placentas donde se determinó el área promedio de la vellosidad, denominada con la sigla APV; el área promedio del capilar, denominada con la sigla APC y la relación de estas dos áreas dadas por un cociente, que se presentó con la sigla $Z$, y cuyas correlaciones están representadas en las gráficas 1, 2 y 3 , donde además de la ubicación de los diferentes puntos que corresponden a los casos estudiados, se encuentra la tendencia de la gráfica dada por la regresión lineal en cada una de ellas.

Como se puede observar el coeficiente de correlación es demasiado pobre $y$ escaso lo que nos indica que no existe correlación entre el grado de madurez o histológico en los casos de alto riesgo estudiados; lo cual podria explicarse o deberse a varios puntos: primero, estadísticamente el universo revisado es pequeño, especialmente para placentas cuyo grado de madurez ecográfico resultó ser 0 y l, pues la mayoría de las placentas quedaron comprendidas entre los grados madurez ecográfica II y III.

Segundo: se trabajó con un reducido rango de edades gestacionales, que como se sabe está comprendido entre las treinta y seis y cuarenta y una semanas, debido a que no entró ninguna paciente al protocolo de trabajo, que se desembarazara por debajo de la trigésima sexta semana.

Por último, existe un punto de vista diferente entre el estudio ecográfico de la placenta y el estudio histopatológico o morfológico de la misma, hecho mucho más significativo en los grados placentarios más inmaduros, debido a que es muy diferente estudiar la textura o 
AFV (WEIt

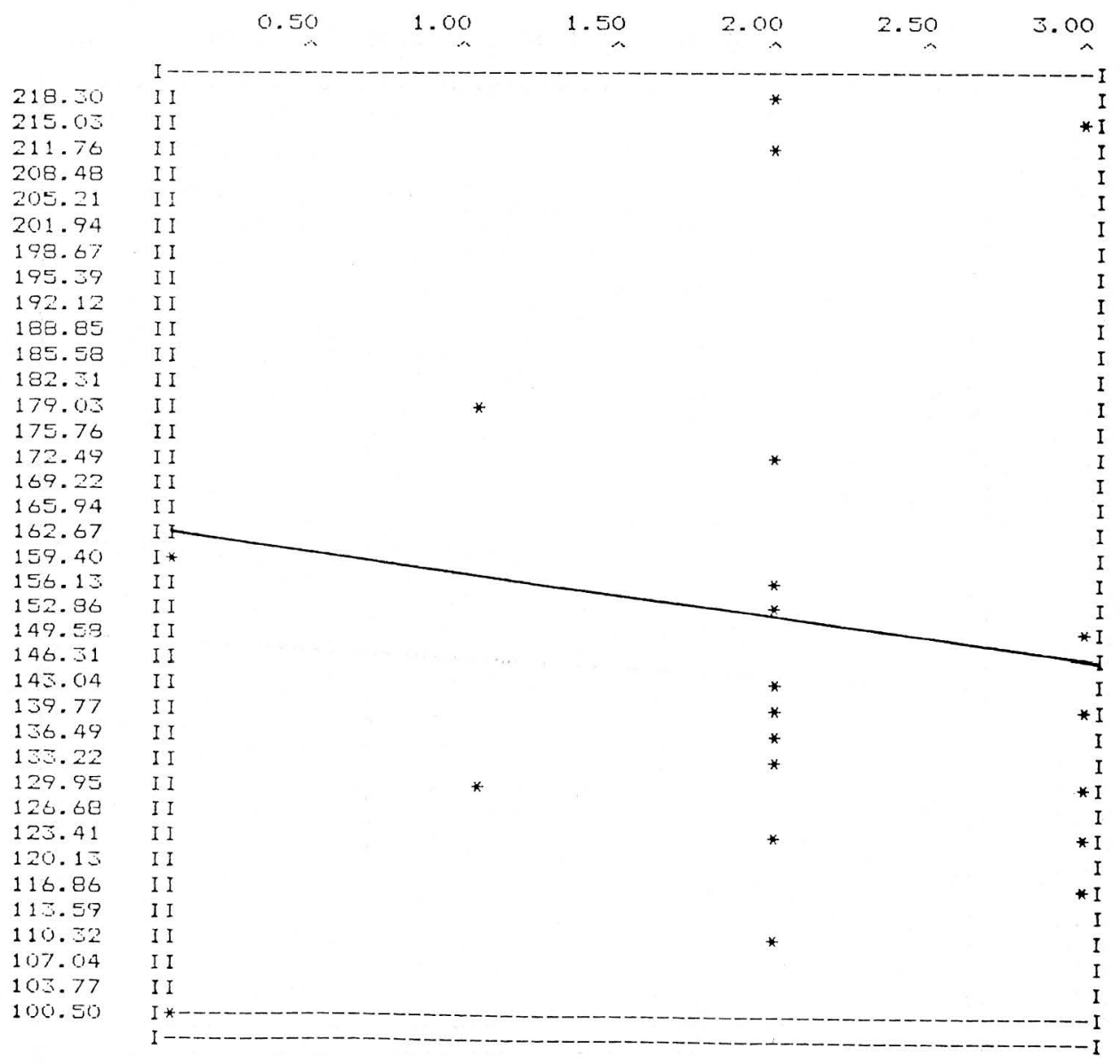

GRAFICA No. 1

substancia del tejido placentario ultrasonográficamente, a ser estudiada histopatológicamente.

También se hicieron correlaciones entre la edad gestacional por amenorrea, denominada con la sigla $E G, y$ diferentes parámetros de desarrollo ecográfico y físicos como el diámetro biparietal del feto, denominada con la sigla DBP; la talla de los recién nacidos dada por la valoración de Usher, denominada con la sigla $\mathrm{U}$, como se observa en las gráficas 4,5 y 6; donde además de la ubicación 

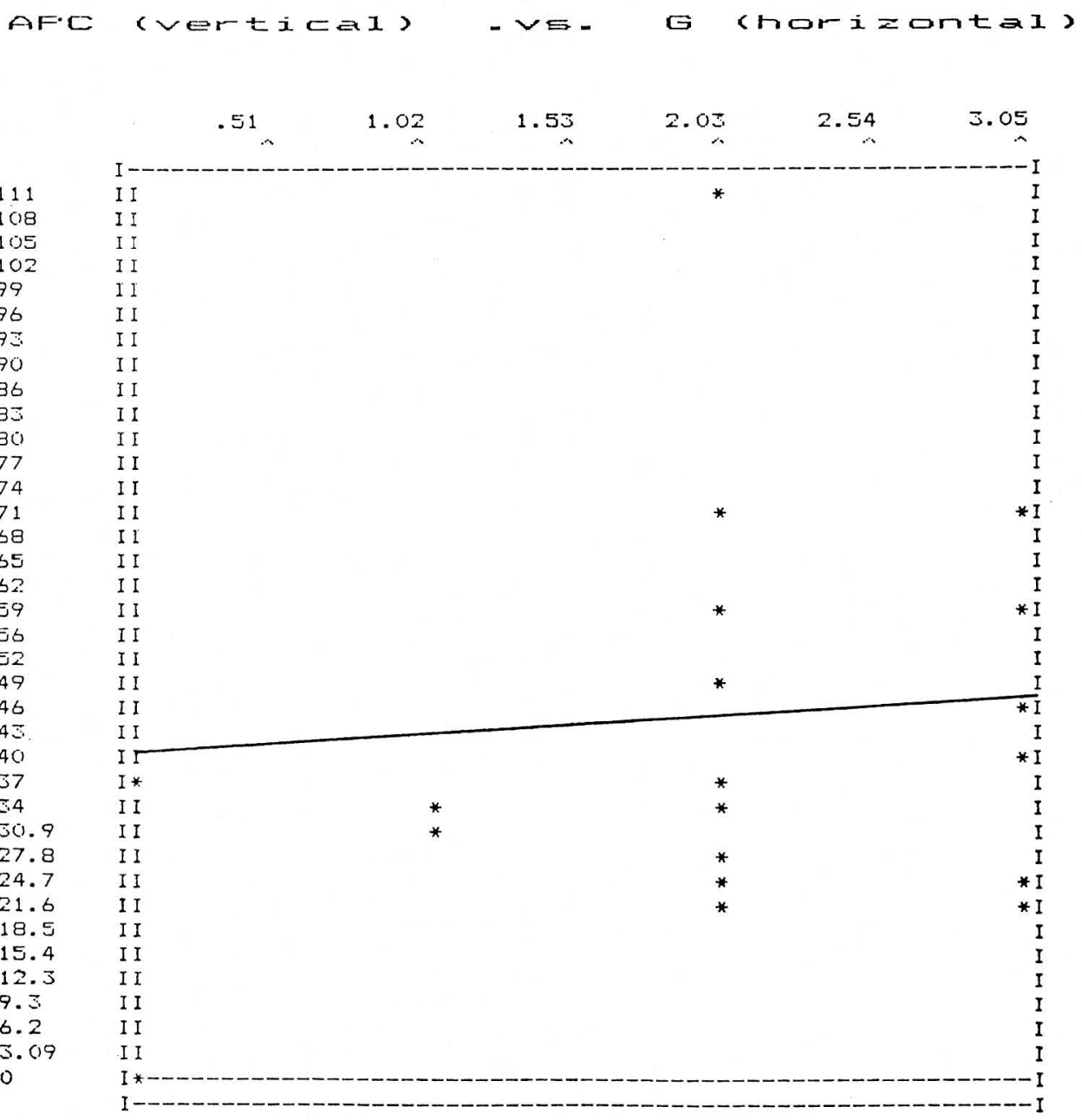

GRAFICA No. 2

en puntos de los diferentes casos correlacionados, se encuentra la tendencia de la gráfica, dada por la regresión lineal de los mismos.

En estas gráficas se ve que existe una asociación discreta entre los factores correlacionados, pero en ningún caso es estadísticamente significativa, o sea que no existe una buena correlación entre los diversos parámetros que se utilizan para determinar la edad gestacional. La correlación menos deficiente fue entre la edad de los recién nacidos valorada por 


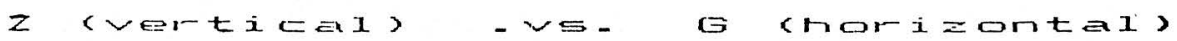

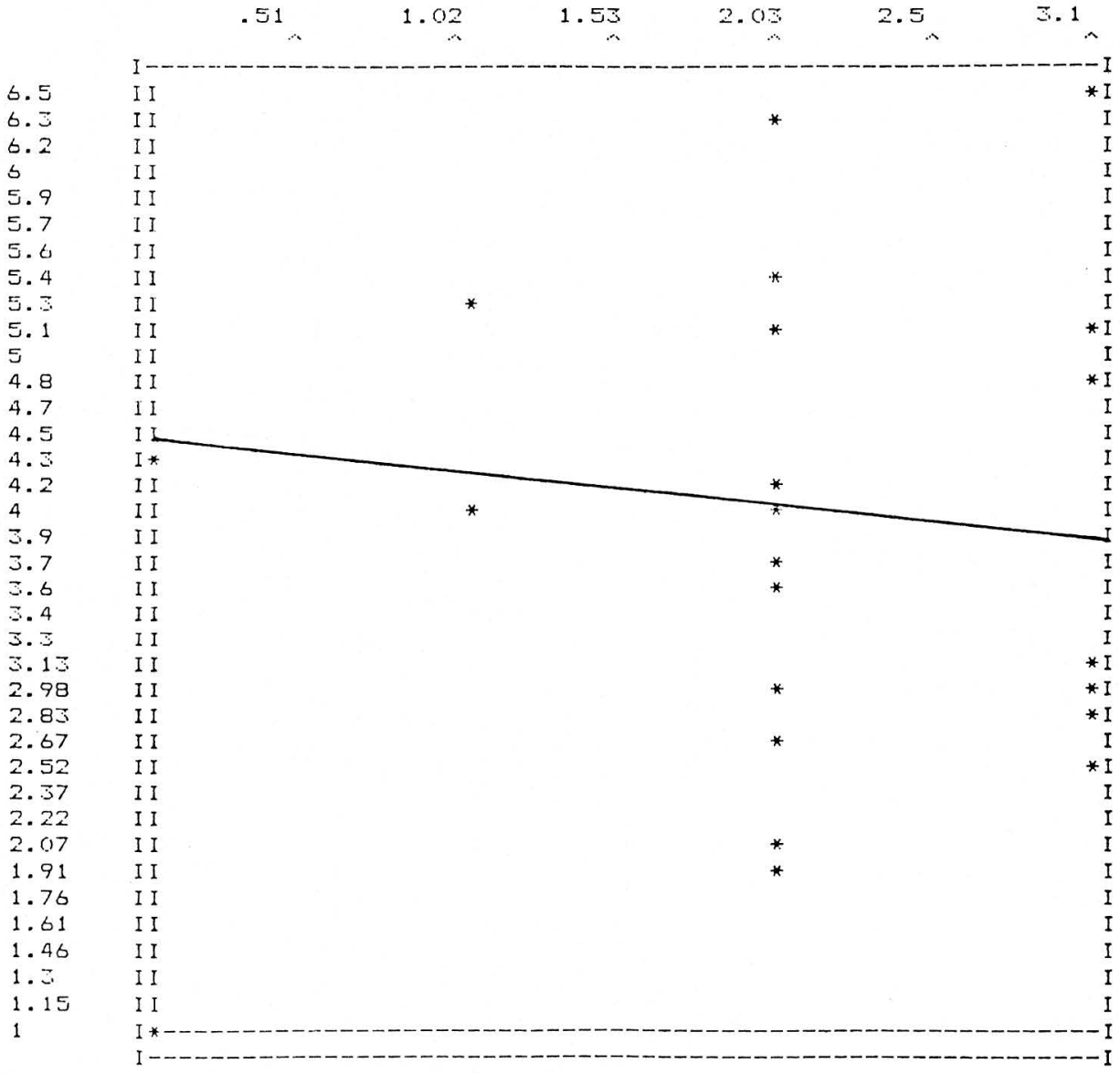

GRAFICA No. 3

los parámetros de Usher (U) y la edad gestacional por amenorrea (EG).

\section{CONCLUSIONES}

Como ninguno de los recién nacidos presentó sindrome de insuficiencia respiratoria, puede decirse que teniendo placentas inmaduras tanto, morfológicamente como ecográficamente no puede predecirse la inmadurez pulmonar en los productos de mujeres con embarazos 
DEP (WERt

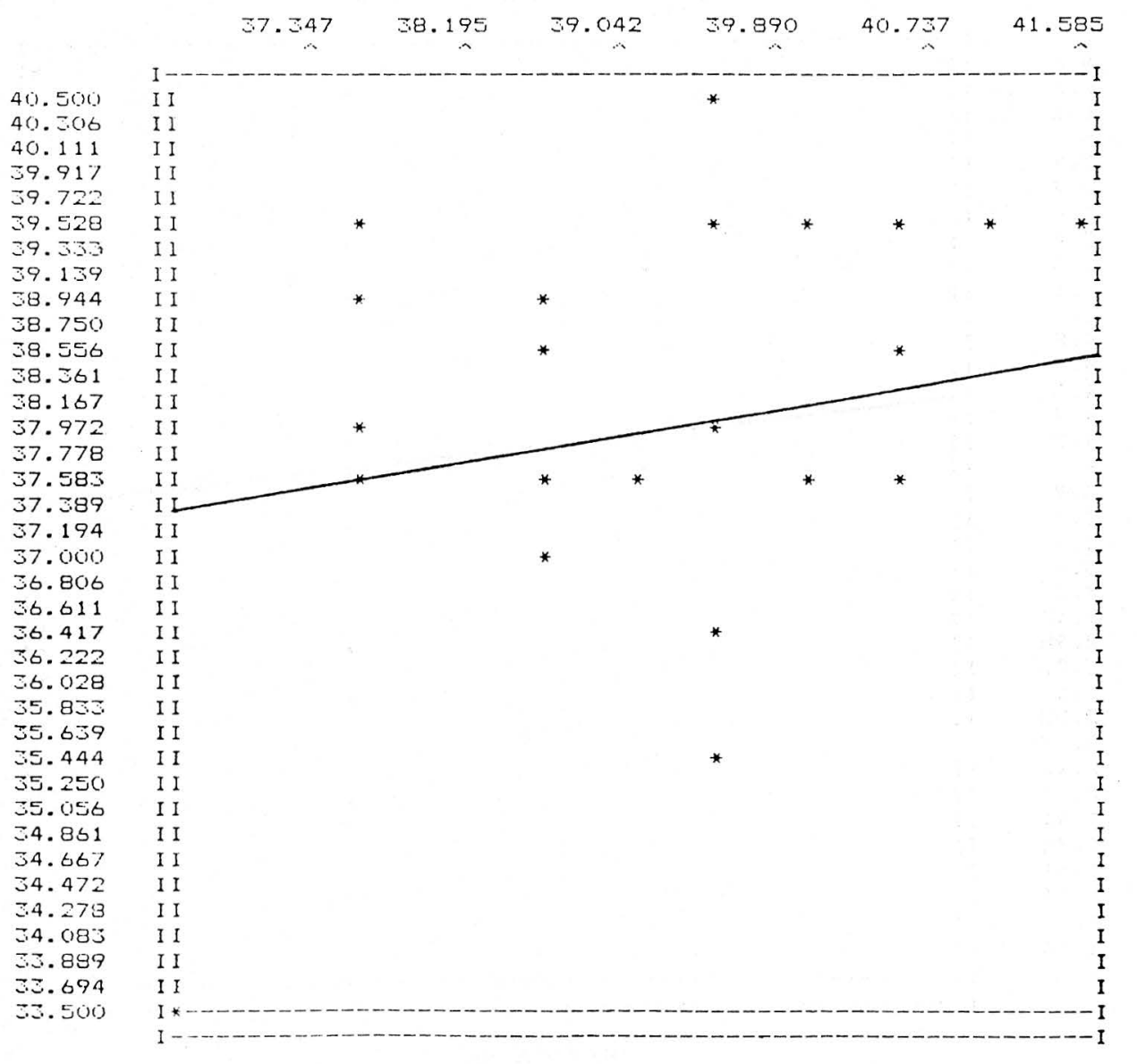

GRAFICA No. 4

de alto riesgo por enfermedad hipertensiva del embarazo o con hipertensión agregada.

Por encima de la semana treinta y seis del embarazo los procesos de madura- ción han alcanzado el máximo grado de desarrollo especialmente en los embarazos de alto riesgo que se acompañan de hipertensión arterial, como en nuestro estudio, y es por esto que las correlaciones no son significativas. 


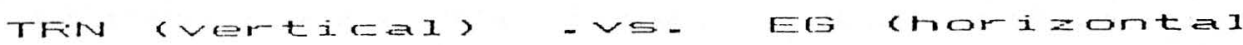

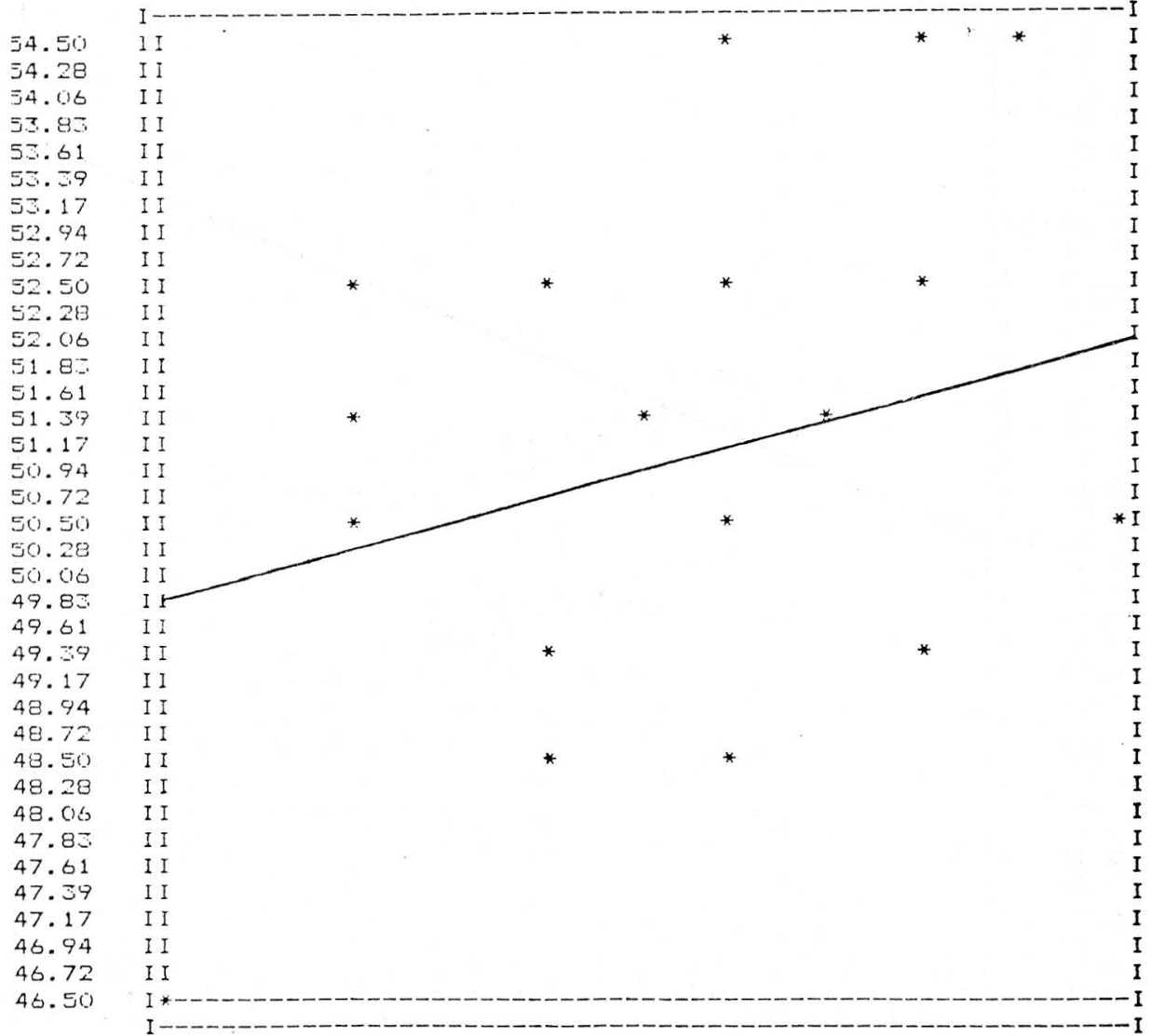

GRAFICA No. 5

En nuestro trabajo no se pudo demostrar correlación adecuada entre la madurez placentaria por Ecografía y la madurez placentaria por histología o morfologia, por lo cual se recomienda para futuros trabajos y estudios en este cam- po, una ampliación en número de casos y rango de semanas, especialmente buscar las mujeres con embarazos de alto riesgo que se desembaracen por una $u$ otra razón, antes de la semana treinta y seis de gestación, para buscar una corre- 
U (vertical) - VE- EG (harizonta1)

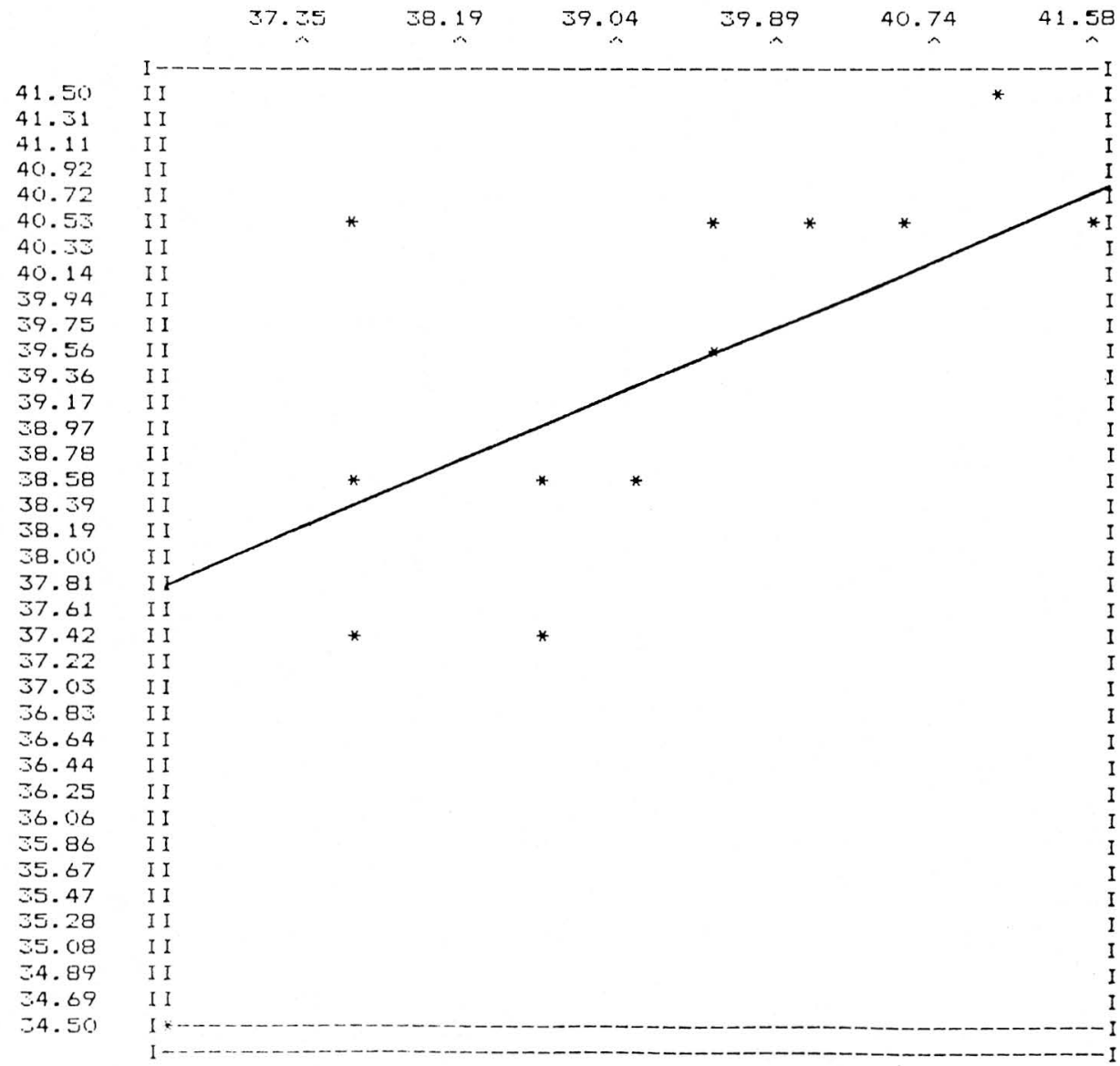

GRAFICA No.6

lación más precisa entre la madurez ecográfica y la madurez morfológica o histológica con el fin de determinar qué validez tiene la valoración ecográfica de la maduración placentaria en los embarazos de al to riesgo.
Creemos que podemos hacer una contribución modificando la clasificación de Grannumy y Cols. para que resulte más objetiva al observador y pueda codificarse, resultando más confiable estadísticamente al ser leídos los parámetros 
de clasificación de madurez y calificarlos con un número progresivo de acuerdo a si existe o no un cambio en la placa corial, la textura o substancia placentaria o en la placa basal.

En cuanto a los cambios de la placa corial, tenemos que para cada grado desde el 0 hasta el $1 \mathrm{II}$ existen cambios notorios y definibles grado a grado, por lo cual a cada uno se le ha calificado con un número progresivo de 1 a 4 respectivamente. En los cambios de la textura o substancia placentaria, no existe ninguna variación estructural entre el grado
I y el grado II, por lo tanto y siguiendo con la progresión numérica a ambos grados le corresponde el número 2 , pues no hay diferencia entre los dos grados, como si hay entre su grado anterior o sea el 0 al cual le corresponde el número I y para el último grado o sea el $|I|$, le corresponde el siguiente número en el orden, el 3. Para los cambios en la placa basal se encontró que no existe ninguna diferencia de esta estructura en los grados 0 y I, lo que obliga a calificarlos con el mismo número con que se inicia la calificación, el I, como para el grado II existen cambios en la estructura, enton-

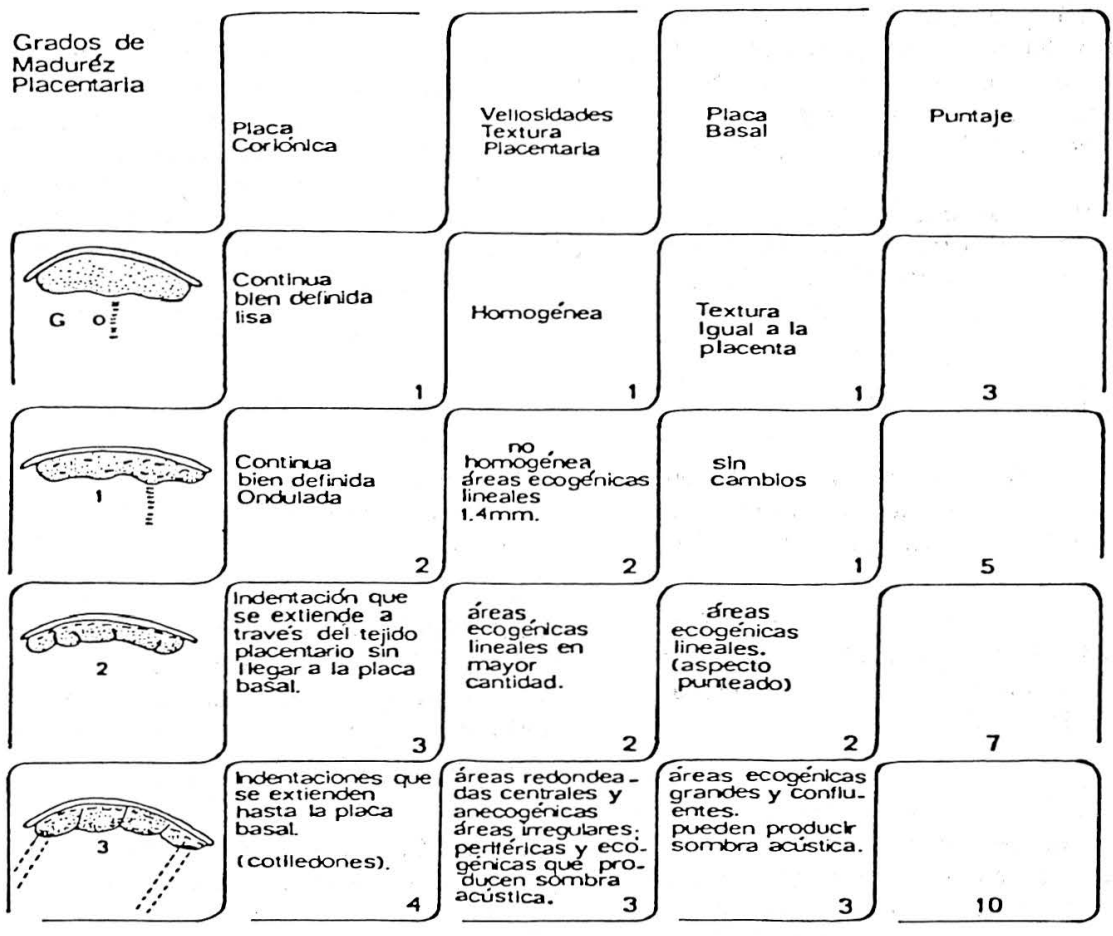

- CUADRO \#2: CLASIFICACION MODIFICADA DE LOS CAMBIOS ECOGRAFICOS EN LA MADUREZ PLACENTARIA. 
ces se califica con el siguiente número, el 2 ; y de igual forma como existe diferencia estructural en el siguiente grado con respecto a su anterior, a este último grado le corresponde el 3.

Como se conocen exactamente los cambios estructurales en cada uno de los grados dados por Grannumy y Cols. en el estudio ecográfico de la madurez placentaria y según se ha explicado anteriormente y cada uno le corresponde un número, no es sino adicionarlos, para poder establecer a que grado corresponde la lectura hecha por el ecografista.

De tal forma que con el sistema propuesto se pasa de la subjetividad a la objetividad en la clasificación ultrasonográfica de la madurez placentaria evitando las variaciones de persona a persona.

En nuestra clasificación propuesta, al grado 0 le corresponde un puntaje hasta de 3; el grado I un puntaje de 5; al grado

\section{BIBLIOGRAFIA}

1. SYMONDS E.M. Estados Hipertensivos en el embarazo. Temas Actuales de Ginecologia y Obstetricia. Interamericana, Dic., 1977.

2. KURTZ A.B. WAPNER R.J. KURTZ R.J., et al. Análisis del diámetro biparietal como un indicador preciso de la edad gestacional. JCU, 198; 8: 319-326.

3. CAMPBELL S. Circunferencia de la cabeza fetal relacionada con la edad gestacional. In: Sanders R. James $A E$, eds. Los principios y práctica de la Ultrasonografía en
II un puntaje hasta de 7 y al grado III un puntaje hasta de 10. (Cuadro No. 2).

\section{COMENTARIO:}

Se revisaron las placentas de 40 pacientes con enfermedad Hipertensiva del embarazo é Hipertensión Arterial Asociada, diagnósticada por los parámetros clínicos usuales; las cuales fueron desembarazadas por encima de la 36o. semana, y a quienes se les había practicado previamente con un tiempo no mayor de 48 horas una Ecografía, mediante la cual se revisaron los patrones de madurez fetal y placentaria. Las placentas fueron analizadas sistemáticamente y minuciosamente tanto macro como microscópicamente pudiéndose establecer una relación de los hallazgos histopatológicos y ecográficos de tal manera que se sugiere una nueva clasificación ecográfica de la madurez placentaria.

Obstetricia y Ginecología, Segunda Edición N. York: Appleton-Century-Crofts, 1980: 454.

4. GRANNUM P.A. BERKOWITZ R.L., HOBBINS J.C. Cambios ultrasonográficos en la madurez placentaria y su relación con la madurez fetal pulmonar. (Am. J. Obstet. Gynecol. 133: 915, 1979).

5. URIBE J.G. Maduración de las vellosidades placentarias. Congreso de Obstet. y Ginecol., 1979. 